\title{
Clinical usefulness of lipid ratios to identify men and women with metabolic syndrome: a cross-sectional study
}

\author{
Danijela Gasevic $^{1 *}$, Jiri Frohlich ${ }^{2,3}$, GB John Mancini ${ }^{4}$ and Scott A Lear ${ }^{5,3}$
}

\begin{abstract}
Background: Waist circumference, a metabolic syndrome (MetSy) criterion, is not routinely measured in clinical practice making early identification of individuals with MetSy challenging. It has been argued that ratios of commonly measured parameters such as lipids and lipoproteins may be an acceptable alternative for identifying individuals with MetSy. The objective of our study was to explore clinical utility of lipid ratios to identify men and women with MetSy; and to explore the association between lipid ratios and the number of MetSy components.

Methods: Men and women ( $N=797)$ of Aboriginal, Chinese, European, and South Asian origin (35-60 years), recruited across ranges of body mass index (BMI), with no diagnosed cardiovascular disease (CVD) or on medications to treat CVD risk factors were assessed for anthropometrics, family history of CVD, MetSy components (waist circumference, blood pressure, glucose, triglycerides (TG), high-density-lipoprotein-cholesterol (HDL-C)), low-density-lipoprotein-cholesterol (LDL-C), nonHDL-C, and health-related behaviours.

Results: Mean levels of lipid ratios significantly increased with increasing number of MetSy components in men and women $(p<0.05)$. After adjustment for age, ethnicity, smoking, alcohol consumption, physical activity, family history of CVD and BMI, (and menopausal status in women), all lipid ratios were associated with the number of MetSy components in men and women (Poisson regression, $\mathrm{p}<0.001$ ). Compared to the rest of the lipid ratios (ROC curve analysis), TG/HDL-C was best able to discriminate between individuals with and without MetSy (AUC $=0.869$ (95\% Cl: 0.830, 0.908) men; $A \cup C=0.872$ (95\% Cl: 0.832, 0.912) women). The discriminatory power of TC/HDL-C and nonHDL-C/HDL-C to identify individuals with MetSY was the same (for both ratios, AUC $=0.793$ (95\% Cl: 0.744, 0.842) men; 0.818 (95\% Cl: 0.772, 0.864) women). Additionally, LDL-C/HDL-C was a good marker for women (AUC $=0.759(95 \% \mathrm{Cl}: 0.706,0.812)$ ), but not for men (AUC $=0.689(95 \% \mathrm{Cl}$ : 0.631, 0.748)). Based on a multiethnic sample, we identified TG/HDL-C cut-off values of 1.62 in men and 1.18 in women that were best able to discriminate between men and women with and without MetSY.
\end{abstract}

Conclusions: Our results indicate that TG/HDL-C is a superior marker to identify men and women with MetSy compared to TC/HDL-C, LDL-C/HDL-C, and nonHDL-C/HDL-C.

Keywords: Metabolic syndrome, Lipid ratios, Triglyceride-to-high-density-lipoprotein-cholesterol, Low-densitylipoprotein-cholesterol-to-high-density-lipoprotein-cholesterol, Non-high-density-lipoprotein-cholesterol-to-highdensity-lipoprotein-cholesterol, and total cholesterol-to-high-density-lipoprotein-cholesterol

\footnotetext{
* Correspondence: dga4@sfu.ca

'Department of Biomedical Physiology and Kinesiology, Simon Fraser University, 2600-515 West Hastings, Vancouver, British Columbia V6B 5K3, Canada

Full list of author information is available at the end of the article
}

\section{() Biomed Central}

(c) 2014 Gasevic et al.; licensee BioMed Central Ltd. This is an Open Access article distributed under the terms of the Creative Commons Attribution License (http://creativecommons.org/licenses/by/4.0), which permits unrestricted use, distribution, and reproduction in any medium, provided the original work is properly credited. The Creative Commons Public Domain Dedication waiver (http://creativecommons.org/publicdomain/zero/1.0/) applies to the data made available in this article, unless otherwise stated. 


\section{Background}

Metabolic syndrome is a cluster of metabolic abnormalities associated with type 2 diabetes [1,2], cardiovascular morbidity and mortality, and all-cause mortality [3,4]. It is alarming that the prevalence of metabolic syndrome is high and on the rise in both developed and developing countries $[5,6]$. Early identification and treatment of individuals with metabolic syndrome is imperative to prevent debilitating consequences associated with its development. Regardless of the approach for diagnosing metabolic syndrome, central obesity, as measured by waist circumference, is one of the main criteria for diagnosing metabolic syndrome [7-10]. However, waist circumference is not routinely measured in primary care [11], which makes early identification of individuals with metabolic syndrome challenging.

It has been argued that ratios of commonly measured parameters such as lipids and lipoproteins may be an acceptable alternative for identifying individuals with metabolic syndrome [12-17]. Evidence shows that lipid ratios perform better than individual lipids in predicting cardiovascular risk [18-21]. Identifying a ratio to serve as a quick and simple tool for identifying individuals at increased cardiometabolic risk may decrease complexity of and increase efficiency in identifying and monitoring those at risk; especially as electronic medical records become more commonplace or if useful biochemical ratios were to be reported in routine laboratory test results. However, research on clinical usefulness of lipid ratios to identify individuals with metabolic syndrome is scarce and mostly limited to specific population groups such as Ghanian [17], Korean [14,16], Spanish [12], and Turkish [13]. Furthermore, some of the available studies researched limited number of ratios [13,17] or provided no cut-offs for lipid ratios to help guide physicians in identifying individuals at cardiometabolic risk [14,15]. Therefore, the objective of this study was to explore the clinical usefulness of lipid ratios to identify men and women with metabolic syndrome. Lipid ratios researched include: total cholesterol-to-high-density-lipoprotein-cholesterol (TC/HDL-C), triglyceride-to-high-density-lipoproteincholesterol (TG/HDL-C), low-density-lipoprotein-cholesterolto-high-density-lipoprotein-cholesterol (LDL-C/HDL-C), and non-high-density-lipoprotein-cholesterol-to-high-densitylipoprotein-cholesterol (nonHDL-C/HDL-C). In addition, given that the number of metabolic syndrome components has been shown to predict incident cardiovascular disease (CVD) and type 2 diabetes [22], we additionally explored the association of lipid ratios with the number of metabolic syndrome components among men and women coming from the ethnically diverse population.

\section{Results}

Participants were equally represented across sexes and ethnicity. Compared to women, men had lower levels of
HDL-C and significantly higher levels of total cholesterol, LDL-C, nonHDL-C, triglycerides, lipid ratios, glucose, blood pressure, and waist circumference; also, alcohol consumption was more prevalent among men than among women (Table 1). Similarly, the average number of metabolic syndrome components and prevalence of metabolic syndrome were higher in men than in women.

Mean levels of lipid ratios were on average higher in men than in women and significantly increased with increasing number of metabolic syndrome components in both men and women (Figure 1, Additional file 1: Table S1). In both men and women, mean levels of lipid ratios in individuals with zero metabolic syndrome components were significantly lower from their counterparts with 1 or more metabolic syndrome components $(\mathrm{p}<0.05)$. In men and women, except for LDL-C/HDL-C ratio, there was a significant difference between mean levels of lipid ratios across number of metabolic syndrome components $(\mathrm{p}<0.05)$. However, in both men and women, no significant difference was found between mean levels of LDL-C/ HDL-C of participants with 3 and those with 4/5 metabolic syndrome components $(p>0.05)$. Furthermore, the results of partial correlation analyses (age and ethnicity adjusted) reveal that in both men and women, compared to the rest of the lipid ratios, TG/HDL-C showed stronger correlations with waist circumference, blood pressure and blood glucose (Additional file 2: Table S2). In addition, the results of Poisson regression analyses indicate that, after adjustment for age, ethnicity, smoking, alcohol consumption, physical activity, family history of CVD and body mass index (BMI) (and menopausal status in women), all lipid ratios were significantly associated with the number of metabolic syndrome components in both men and women (Table 2).

Using ROC curve analyses, we plotted sensitivity over 1-specificity for each of the ratios in both men and women (Figure 2). Compared to the rest of the lipid ratios, TG/HDL-C was best able to discriminate between apparently healthy men and women with and without metabolic syndrome (AUC $=0.869$ (95\% CI: 0.830, 0.908) for men; $\mathrm{AUC}=0.872$ (95\% CI: 0.832, 0.912) for women). The discriminatory power of TC/HDL-C and nonHDL-C/ HDL-C to identify individuals with metabolic syndrome was the same (for both ratios, AUC $=0.793$ (95\% CI: $0.744,0.842)$ for men; 0.818 (95\% CI: $0.772,0.864)$ for women). In addition, based on Hosmer and Lemeshow's criteria [23] LDL-C/HDL-C was an "acceptable" marker to discriminate between women with and without metabolic syndrome (AUC $=0.759$ (95\% CI: 0.706, 0.812)), however this was not the case for men (AUC $=0.689$ (95\% CI: $0.631,0.748)$ ). In both men and women, the analysis that tested the statistical significance of the differences between areas under the curve revealed significant difference between areas under the curves for all the pairs of lipid 
Table 1 Distribution of risk factors in men and women

\begin{tabular}{|c|c|c|c|}
\hline & Men 380 & Women 417 & $p$ value \\
\hline Age (years) & $46.8 \pm 8.7$ & $47.5 \pm 8.9$ & 0.241 \\
\hline Ethnicity & & & 0.704 \\
\hline Aboriginal & $81(21.3 \%)$ & $98(23.5 \%)$ & \\
\hline Chinese & $100(26.3 \%)$ & $118(28.3 \%)$ & \\
\hline European & $97(25.5 \%)$ & $99(23.7 \%)$ & \\
\hline South Asian & $102(26.8 \%)$ & $102(24.5 \%)$ & \\
\hline Current smokers & $44(11.6 \%)$ & $35(8.4 \%)$ & 0.133 \\
\hline Current consumers of alcohol & $128(33.7 \%)$ & $98(23.5 \%)$ & 0.001 \\
\hline Physical activity (min/week) & $226(100,447)$ & $208(95,424)$ & 0.269 \\
\hline Family history of cardiovascular disease & $168(44.2 \%)$ & $187(44.8 \%)$ & 0.857 \\
\hline Waist circumference $(\mathrm{cm})$ & $92.6 \pm 11.2$ & $85.1 \pm 12.2$ & $<0.001$ \\
\hline Body mass index $\left(\mathrm{kg} / \mathrm{m}^{2}\right)$ & $27.6 \pm 4.3$ & $27.3 \pm 5.3$ & 0.498 \\
\hline $\mathrm{TC}(\mathrm{mmol} / \mathrm{L})$ & $5.25 \pm 0.97$ & $5.23 \pm 1.03$ & 0.776 \\
\hline $\mathrm{HDL}-\mathrm{C}(\mathrm{mmol} / \mathrm{L})$ & $1.13 \pm 0.29$ & $1.43 \pm 0.35$ & $<0.001$ \\
\hline LDL-C (mmol/L) & $3.30 \pm 0.85$ & $3.17 \pm 0.92$ & 0.046 \\
\hline $\mathrm{TC} / \mathrm{HDL}-\mathrm{C}$ & $4.91 \pm 1.46$ & $3.86 \pm 1.23$ & $<0.001$ \\
\hline LDL/HDL-C & $3.07 \pm 1.01$ & $2.35 \pm 0.91$ & $<0.001$ \\
\hline nonHDL-C & $4.12 \pm 0.99$ & $3.80 \pm 1.05$ & $<0.001$ \\
\hline nonHDL-C/HDL-C & $3.81(2.93,4.63)$ & $2.68(2.01,3.45)$ & $<0.001$ \\
\hline TG & $1.48(0.99,2.22)$ & $1.17(0.83,1.63)$ & $<0.001$ \\
\hline $\mathrm{TG} / \mathrm{HDL}-\mathrm{C}$ & $1.34(0.82,2.24)$ & $0.86(0.54,1.22)$ & $<0.001$ \\
\hline Systolic BP (mmHg) & $117(110,124)$ & $115(106,126)$ & 0.025 \\
\hline Diastolic BP (mmHg) & $79.2 \pm 9.7$ & $75.5 \pm 9.2$ & $<0.001$ \\
\hline Glucose (mmol/L) & $5.30(5.00,5.60)$ & $5.10(4.80,5.40)$ & $<0.001$ \\
\hline With MetSy & $119(31.3 \%)$ & $104(24.9 \%)$ & 0.045 \\
\hline Number of MetSy components & $1.84 \pm 1.40$ & $1.63 \pm 1.22$ & 0.026 \\
\hline
\end{tabular}

Categorical variables presented as $\mathrm{n}(\%)$, and sex differences were explored using Chi-square test. Continuous variables presented as mean \pm SD if normally distributed or median $(25 \%, 75 \%)$ if skewed. Sex differences explored using t-test and Mann U Whitney test for normally distributed and skewed data, respectively. TC - total cholesterol, HDL-C - high-density lipoprotein cholesterol, LDL-C - low-density lipoprotein cholesterol, TC/HDL-C - ratio of TC and HDL-C, TG - triglycerides, TG/HDL-C - ratio of TG and HDL-C, nonHDL-C/HDL - ratio of nonHDL-C and HDL-C, LDL-C/HDL-C - ratio of LDL-C and HDL-C, MetSy - metabolic syndrome.

ratios (Figure 2). The exception is the pair of TC/HDL-C and nonHDL-C/HDL-C for which AUCs were the same. Lipid ratio cut-off values, with their respective sensitivities and specificities, for identifying individuals with metabolic syndrome are provided in Table 3.

\section{Discussion}

The purpose of this study was to explore the clinical utility of lipid ratios to identify men and women with metabolic syndrome. Additionally we explored the association between lipid ratios and number of metabolic syndrome components. Our results indicate that increases in lipid ratios are significantly associated with increase in the number of metabolic syndrome components in both men and women after adjusting for age, ethnicity, smoking status, alcohol consumption, physical activity, family history of CVD, and BMI (and menopausal status in women).
Compared to TC/HDL-C, LDL-C/HDL-C, and nonHDLC/HDL-C, TG/HDL-C was shown to be a better clinical marker to discriminate between individuals with and without metabolic syndrome. These results were consistent for men and women.

The results of our study are in line with those based on Spanish, Korean, and Japanese populations where increases in lipid ratios were shown to be associated with increase in the number of metabolic syndrome components $[12,14,15]$. Our study extends the previous findings by reporting the positive association between lipid ratios and the number of metabolic syndrome components in a multiethnic population; this relationship persisted after adjusting for ethnicity and other factors known to be associated with metabolic syndrome.

The observed strong relationship between lipid ratios and the number of metabolic syndrome components 


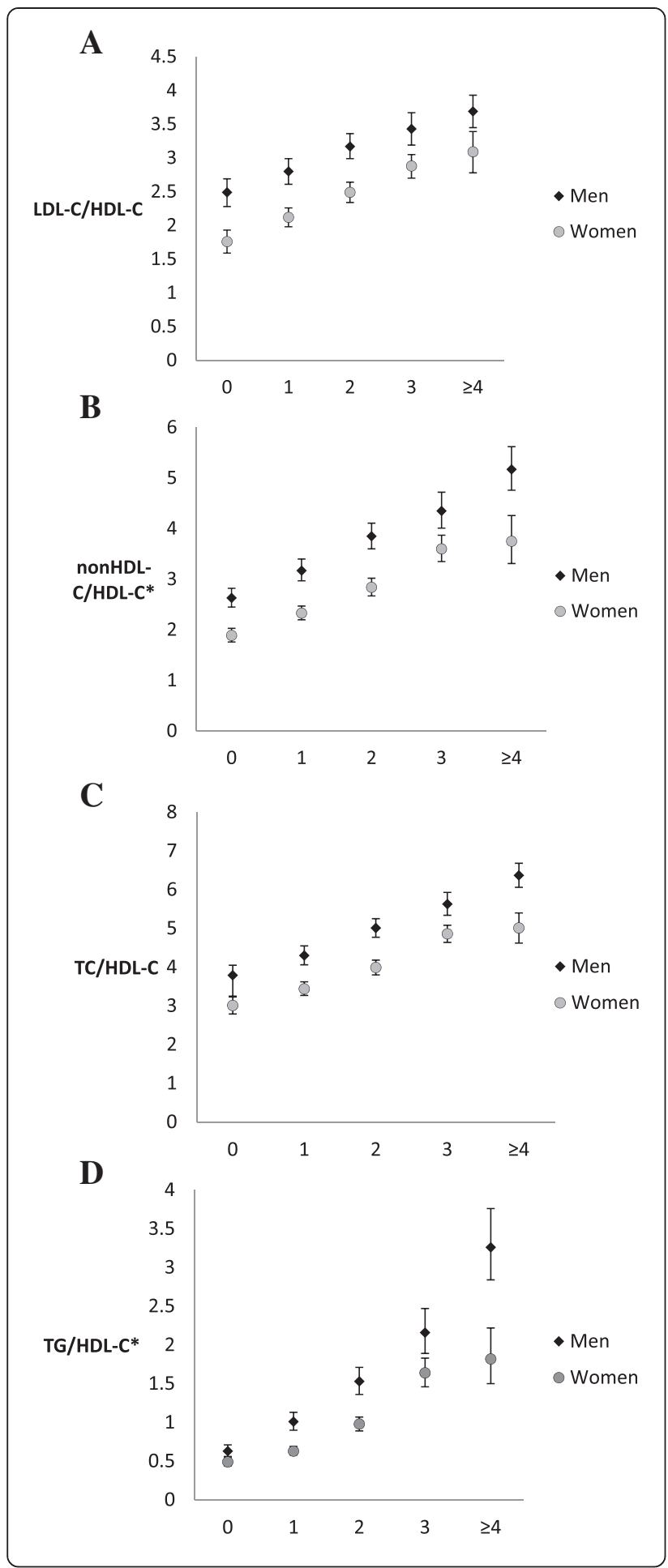

Figure 1 Mean levels of lipid ratios across number of metabolic syndrome components in men and women (1A, 1B, 1C and 1D). All means adjusted for age and ethnicity and presented as mean (95\% CI). * geometric means. Bonferroni-corrected pairwise comparisons ( 0 vs. $1 ; 0$ vs. $2 ; 0$ vs. $3 ; 0$ vs. $\geq 4 ; 1$ vs. $2 ; 1$ vs. $3 ; 1$ vs. $\geq 4 ; 2$ vs. $3 ; 2$ vs. $\geq 4$; and 3 vs. $\geq 4$ ): Men: Except for the pair 3 vs. $\geq 4$ for LDL-C/HDL-C, all other pairwise comparisons of means of TC/HDL-C, TG/HDL-C, LDL-C/HDL-C and nonHDL-C/HDL-C across the number of metabolic syndrome components were significant at $p<0.05$. Women: Except for the pair 3 vs. $\geq 4$, all other pairwise comparisons of means of TC/HDL-C, TG/HDL-C, LDL-C/HDL-C and nonHDL-C/HDL-C across the number of metabolic syndrome components were significant at $p<0.05$.

TG/HDL-C: triglyceride-to-high-density-lipoprotein-cholesterol, TC/HDL-C: total cholesterol-to-high-density-lipoprotein-cholesterol, LDL-C/HDL-C: low-density-lipoprotein-cholesterol-to-high-density-lipoproteincholesterol, nonHDL-C/HDL-C: non-high-density-lipoprotein-cholesterolto-high-density-lipoprotein-cholesterol.

may suggest lipid ratios as potential simple tools for early identification of individuals with constellation of cardiometabolic abnormalities. However, the actual lipid ratio cut-offs to guide clinicians in identifying individuals with metabolic syndrome are rarely reported. The studies available looked at a limited number of lipid ratios and pertained to specific groups such as Spanish population [12] or a population of Ghanian women [17]. We extend the available findings by reporting lipid ratio cut-off values for identifying men and women with metabolic syndrome. According to our results, in both men and women, TG/HDL-C was shown to be a better marker to identify individuals with metabolic syndrome compared to $\mathrm{TC} /$ HDL-C, LDL-C/HDL-C, and nonHDL-C/HDL-C. Based on a multiethnic sample, we identified TG/HDL-C cut-off values of 1.62 in men and 1.18 in women that were best able to discriminate between men and women with and without metabolic syndrome. These numbers were comparable but slightly lower than those reported by Cordero et al. [12] who clinically assessed a large working population of Spanish men and women and reported TG/HDL-

Table 2 The association between lipid ratios and the number of metabolic syndrome components

\begin{tabular}{lll}
\hline & Men & Women \\
& $\operatorname{Exp}(\mathbf{B})(\mathbf{9 5 \%} \mathbf{C l})$ & $\operatorname{Exp}(\mathbf{B})(\mathbf{9 5 \%} \mathbf{C l})$ \\
\hline TC/HDL-C & $1.262(1.197,1.330)$ & $1.278(1.204,1.355)$ \\
In_TG/HDL-C & $1.875(1.680,2.092)$ & $1.797(1.603,2.016)$ \\
LDL-C/HDL-C & $1.236(1.147,1.332)$ & $1.358(1.243,1.483)$ \\
In_nonHDL-C/HDL-C & $2.861(2.258,3.626)$ & $2.633(2.109,3.286)$
\end{tabular}

All Poisson regression models adjusted for age, ethnicity, smoking, alcohol consumption, physical activity, family history of cardiovascular disease, and BMI. Models for women additionally adjusted for menopause status. All models significant at $\mathrm{p}<0.001$. TC/HDL-C - ratio of total cholesterol and high-density lipoprotein cholesterol, TG/HDL-C - ratio of triglycerides and high-density lipoprotein cholesterol, nonHDL-C/HDL - ratio of non-high-density lipoprotein cholesterol and high-density lipoprotein cholesterol, LDL-C/HDL-C ratio of low-density lipoprotein cholesterol and high-density lipoprotein cholesterol. 

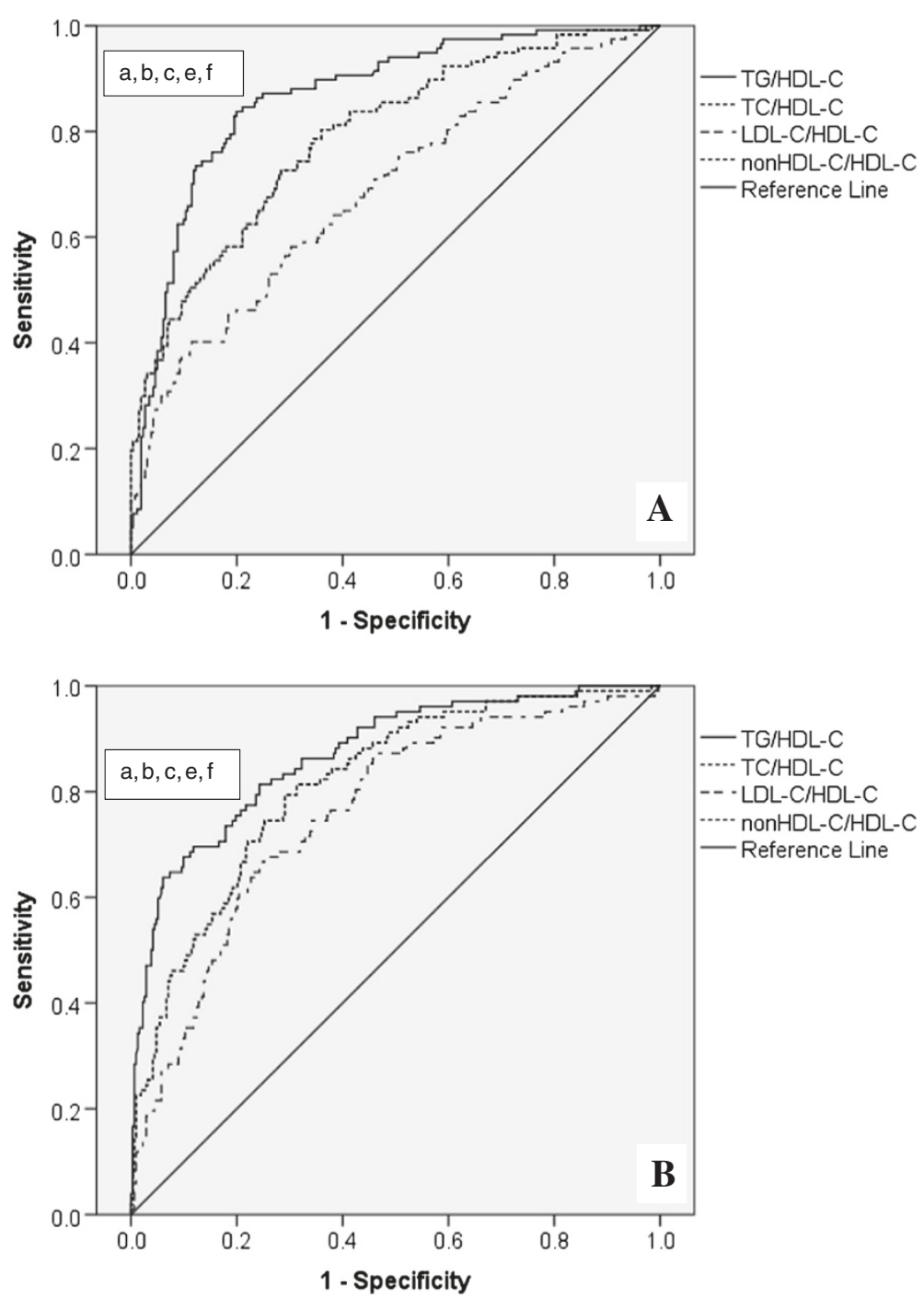

Figure 2 Receiver operating characteristic curves for evaluating the usefulness of lipid ratios to identify men (A) and women (B) with metabolic syndrome. The diagonal line indicates a test with an area under the receiver operating characteristic curve of 0.5 . The areas under the curve for TC/HDL-C and nonHDL-C/HDL-C overlapped, as the discriminatory power of TC/HDL-C and nonHDL-C/HDL-C to identify individuals with metabolic syndrome was the same. The difference between areas under the curve $(p \leq 0.01)$ : ${ }^{\mathrm{a} T G} / \mathrm{HDL}-\mathrm{C}$ vs. TC/HDL-C. ${ }^{\mathrm{b}} \mathrm{TG} / \mathrm{HDL}-\mathrm{C}$ vs. nonHDL-C/HDL-C. ${ }^{C} \mathrm{TG} / \mathrm{HDL}-\mathrm{C}$ vs. LDL-C/HDL-C. ${ }^{\mathrm{d} T C / H D L-C}$ vs. nonHDL/HDL-C. ${ }^{\mathrm{T}} \mathrm{TC}$./HDL-C vs. LDL-C/HDL-C. ${ }^{\mathrm{f}}$ nonHDL-C/HDL-C vs. LDL-C/HDL-C. TG/HDL-C: triglyceride-to-high-density-lipoprotein-cholesterol, TC/HDL-C: total cholesterol-to-high-density-lipoprotein-cholesterol, LDL-C/HDL-C: low-density-lipoprotein-cholesterol-to-high-density-lipoprotein-cholesterol, nonHDL-C/HDL-C: non-high-density-lipoprotein-cholesterol-to-highdensity-lipoprotein-cholesterol.

Table 3 Results of receiver-operating curve analyses featuring the thresholds of lipid ratios with their respective sensitivities and specificities for diagnosing metabolic syndrome in men and women

\begin{tabular}{lll}
\hline & Men & Women \\
\hline Lipid ratio & Criterion value (Sensitivity, specificity) & Criterion value (Sensitivity, specificity) \\
TG/HDL-C & $1.62(84.0 \%, 80.1 \%)$ & $1.18(70.2 \%, 88.2 \%)$ \\
TC/HDL-C & $5.05(73.1 \%, 71.6 \%)$ & $3.91(81.7 \%, 68.7 \%)$ \\
LDL-C/HDL-C* & $3.79(40.2 \%, 88.5 \%)$ & $2.53(67.6 \%, 74.8 \%)$ \\
nonHDL-C/HDL-C & $4.05(73.1 \%, 71.6 \%)$ & $2.91(79.8 \%, 70.9 \%)$ \\
\hline
\end{tabular}

*According to the AUC, LDL-C/HDL-C showed to be a good marker of metabolic syndrome in women but not in men. 
$C$ values of 2.75 and 1.65 as cut-offs for identifying men and women with metabolic syndrome, respectively. The observed differences in cut-offs reported in the above mentioned study and the ones reported in this study are most likely due to a difference in population groups studied.

It has recently been reported that TG/HDL-C predicts the development of CVD as effectively as the diagnosed metabolic syndrome [24]. Similarly, TG/HDL-C was found to predict coronary heart disease and CVD mortality as well as or even better than does metabolic syndrome in men [25]. However, the detection of an elevated TG/HDL-C is not meant to replace metabolic syndrome diagnosis in clinical practice; and it should rather be considered as a simple tool to quickly identify patients at increased cardiometabolic risk for whom further risk evaluation and clinical intervention are needed [25]. Indeed, it has recently been reported in the study in young adults that, compared to metabolic syndrome diagnostic, TG/HDL-C may be able to identify a greater number of individuals at risk; however a use of a metabolic syndrome diagnostics approach identifies individuals with an accentuated cardiometabolic risk profile [26]. While metabolic syndrome diagnosis may provide more comprehensive approach to identifying present cardiometabolic risk factors in individuals, this diagnostics is challenged by the fact that waist circumference, one of the integrative components of metabolic syndrome, is not commonly measured. Indeed, it has recently been reported that WC is routinely measured by only $6 \%$ of primary care physicians [11]. If translated, it may mean that more than $90 \%$ of primary care physicians would not be able to diagnose individuals with metabolic syndrome given that one of its components is not routinely measured. In contrast, both TG and HDL-C are routinely measured in clinical practice, and TG/HDL-C could be readily calculated (or provided by the laboratory) to serve as a quick tool to identify men and women at increased cardiometabolic risk. This approach to use TG/HDL-C to identify individuals at risk for whom further care is needed may reduce time and complexity for initial diagnosis of people with constellation of cardiometabolic abnormalities.

Other research groups have also proposed TG/HDL-C as a potential, simple tool to identify patients at increased risk for CVD. The evidence shows a strong association between TG/HDL-C and insulin resistance as measured by glucose clamp [27,28], modified insulin suppression test [29], and homoeostasis model assessment index $[29,30]$. This association was also found to be independent of waist circumference [31]. Further, others found TG/HDL-C to be an independent predictor of future type 2 diabetes mellitus [25,32] and its related microvascular complications [33]; coronary heart disease
[34,35]; major cardiovascular events including overall death, myocardial infarction, and unstable angina that required revascularization [36] and those including angina pectoris, myocardial infarction, myocardial revascularization, and fatal or nonfatal stroke [37]; and first coronary event irrespective of BMI [38]. The strong relationship between TG/HDL-C and CVD may be founded in the atherogenic properties of TG/HDL-C. It has been reported that increase in TG/HDL-C is significantly associated with decrease in LDL particle size and increase in fractional esterification rates of cholesterol in plasma depleted of apoB-lipoproteins; hence the proposed name for TG/HDL-C being atherogenic index of plasma [39]. In light of available evidence, including the results of our study, it seems reasonable to conclude that TG/HDL-C may serve as a useful marker to identify individuals at increased risk of CVD.

Several limitations to our study should be considered. This study is a cross-sectional design, thus longitudinal studies are needed to explore whether the association between TG/HDL-C and metabolic syndrome persists or changes over time in men and women. Further, the M-CHAT participants were recruited across a range of BMI, so this study may not be representative of the general population. However, recruiting people across ranges of BMI allows for the opportunity to explore the associations between lipid ratios and metabolic syndrome in the population with a range of body sizes. Also, the results of our analyses indicate a significant association between lipid ratios and number of metabolic syndrome components independent of BMI. Moreover, our analyses were adjusted for age, ethnicity, smoking, status, alcohol consumption, physical activity, family history of CVD and BMI (and menopausal status in women) but not for diet known to influence plasma TG levels. Of importance, TG/ HDL-C cut-points for identifying individuals at risk may vary across ethnicity and race [40,41]. Also, TG/HDL-C was shown not to be a reliable risk marker in individuals of South Asian [41] and African American origin [42,43]. While statistical power did not allow us to additionally stratify our analyses by ethnicity, given the established ethnic differences in the way body fat is accumulated $[44,45]$ and ethnic differences in cardiometabolic risk $[46,47]$, we believe that further research featuring ethnicspecific analyses is warranted.

\section{Conclusions}

In conclusion, our study shows strong positive association between lipid ratios and metabolic syndrome in apparently healthy men and women (without CVD and not on CVD-related medications) drawn from an ethnically diverse population. TG/HDL-C appeared to be a superior marker compared to TC/HDL-C, LDL-C/HDL-C, and nonHDL-C/HDL-C, and it is useful for identifying 
both men and women with metabolic syndrome. Namely, TG/HDL-C readings of 1.62 or greater in men and 1.18 or greater in women can help primary care physicians easily identify individuals at increased risk for CVD. Early identification of individuals at risk would allow for an early implementation of lifestyle and medication strategies, while TG/HDL-C could be further used to evaluate the success of such strategies.

\section{Methods}

Apparently healthy men and women were recruited as part of the Multi-Cultural Community Health Assessment Trial (M-CHAT) designed to compare body fat distribution of Aboriginal, Chinese, and South Asian populations to that of a European population and explore how fat distribution relates to CVD risk factors [48]. Details on recruitment of study participants have already been published [48]. In brief, eligible participants were apparently healthy individuals between ages of 30 and 65 of Aboriginal, Chinese, European and South Asian origin residing in the Vancouver mainland. To ensure a range of body fat mass across groups, participants were recruited from the following BMI $\left(\mathrm{kg} / \mathrm{m}^{2}\right)$ ranges: 18.5-24.9 (low range), 25.029.9 (middle range), and 30 and over (upper range). Given that it was challenging to identify Aboriginal men with a BMI of less than 25, Aboriginal men of any BMI were recruited. Similarly, due to a difficulty in identifying Chinese individuals with a BMI of $30 \mathrm{~kg} / \mathrm{m}^{2}$ and higher, the target for the upper BMI range was changed to a BMI of 28 and higher. Individuals with a fluctuation in weight that was greater than $2.5 \mathrm{~kg}$ three months prior to the assessment date, and those with diagnosed CVD or on medications to treat CVD risk factors, were not eligible for the study. Ethnicity was self-reported. All study participants provided informed consent for the study, and the study was approved by the Simon Fraser University Research Ethics Board.

All participants were assessed for socio-demographics and risk factors. Following the standard protocols for blood sample collection, shipment, and processing, fasting blood samples were collected from each participant at the research site and assessed for lipids and glucose at St. Paul's Hospital in Vancouver, Canada. Glucose, TC, TG, and HDL-C were determined using standard protocols by ADVIA 1650 analyzer (Bayer Health Care, LLC., New Jersey, USA). Friedwald formula was used to calculate LDL-C [49]. Height and weight were measured, and BMI was calculated as weight in kilograms divided by height in metres squared. Waist circumference represented an average of two measurements taken upon expiration at the mid point between the low rib margin and iliac crest. Blood pressure was recorded as mean of 5 measurements taken after 10 minutes seated rest in the left arm using BpTRU model BPM-200 oscillometric office blood pressure monitor (VSM MedTech Ltd., Coquitlam, British Columbia). Smoking status (current smokers/non-smokers), alcohol consumption (currently consume alcohol/do not consume alcohol) and family history of CVD (yes/no) were determined by self-report. Women self-reported their menopausal status by choosing from the following options (women were provided a definition for each of the options): pre-menopausal (regular menstrual cycles), peri-menopausal (menopausal transition with symptoms such as menstrual irregularity), post-menopausal (end of menstrual cycles, at least 12 months since the last menstrual period), or hysterectomy (surgical removal of the uterus). Menopausal status variable used as a confounder in regression analyses consisted of two categories, pre-menopausal (pre-menopausal and peri-menopausal) and menopausal (post-menopausal and hysterectomy). Physical activity was measured using the Modifiable Physical Activity questionnaire previously used in multi-ethnic populations and determined by self-report [50,51]. A person would qualify as having metabolic syndrome if he/she had three out of 5 risk factors present (hypertension, hypertriglyceridaemia, lowered HDL-C, hyperglycaemia, and central obesity) using the most recent harmonized definition of the metabolic syndrome where ethnic specific thresholds for waist circumference were used [10].

\section{Statistical analyses}

Only participants with complete data for all metabolic syndrome components were included to the study $(\mathrm{N}=797)$. Considering significant sex differences in body fat distribution and cardiometablic risk [52-54], all statistical analyses were performed separately for men and women. Categorical variables were presented as counts and percentages, and differences between men and women were explored using Chi-square test. Continuous variables were presented as means \pm standard deviation (SD) if normally distributed, or medians with $25^{\text {th }}$ and $75^{\text {th }}$ percentiles if not normally distributed. Differences between men and women in the distribution of continuous variables were explored using t-test and Mann U Whitney test for normally distributed and skewed data, respectively.

Age and ethnicity adjusted levels of lipid ratios across the number of metabolic syndrome components were determined using general linear modelling (Bonferronicorrected post hoc comparisons). In our apparently healthy multiethnic study population, there was a limited number of participants with $4(n=66)$ and $5(n=18)$ metabolic syndrome components; therefore, we combined these two categories. Poisson regression analysis was used to explore the association between lipid ratios and the number of metabolic syndrome components. All models were adjusted for age, ethnicity, smoking, alcohol consumption, physical activity, family history of CVD 
and BMI. Models for women were additionally adjusted for menopausal status.

The clinical utility of lipid ratios to identify individuals with metabolic syndrome was explored using receiveroperating characteristic curve (ROC) analysis. Plots of sensitivity (true positives) versus 1 minus specificity (false positives) were constructed in both men and women for each lipid ratio. Area under the curve (AUC) was calculated to explore which lipid ratio showed highest accuracy in predicting metabolic syndrome. AUC is a measure of discrimination, and AUC of $0.5,0.6 \leq \mathrm{AUC}<7,7 \leq \mathrm{AUC}<$ $0.8,0.8 \leq \mathrm{AUC}<0.9$, and $\geq 0.9$ corresponds to no discrimination, poor, acceptable, excellent, and outstanding discrimination, respectively [23]. Optimal cut-off point of each lipid ratio to identify individuals with metabolic syndrome corresponded to a maximum value of Youden index that was calculated as sensitivity + specificity -1 [55,56]. Additionally, the statistical significance of the difference between areas under the curves was tested using the methods of De Long et al. [57]. Analyses were performed using Statistical Package for Social Sciences (SPSS) version 19 except for the comparisons of ROC curves that were performed using MedCalc statistical software. $\mathrm{P}$ values of less than 0.05 were considered statistically significant.

\section{Additional files}

Additional file 1: Table S1. Mean levels (95\% Cl) of lipid ratios across increasing number of metabolic syndrome components.

Additional file 2: Table S2. Partial correlations (age and ethnicity adjusted) between lipid ratios and metabolic syndrome components in men (A) and women (B).

\section{Abbreviations}

AUC: Area under the curve; BMI: Body mass index; CVD: Cardiovascular disease; LDL-C/HDL-C: Low-density-lipoprotein-cholesterol-to-high-densitylipoprotein-cholesterol; M-CHAT: Multi-Cultural Health Assessment Trial; nonHDL-C/HDL-C: non-high-density-lipoprotein-cholesterol-to-high-densitylipoprotein-cholesterol; ROC: Receiver-operating characteristic curve; SD: Standard deviation; SPSS: Statistical Package for Social Sciences; TC/HDL-C: Total cholesterol-to-high-density-lipoprotein-cholesterol; TG/HDL-C: Triglyceride-to-high-density-lipoprotein-cholesterol.

\section{Competing interests}

The authors declare that they have no competing interests.

\section{Authors' contribution}

DG designed the study, performed statistical analyses and data interpretation and wrote the manuscript. GBJM participated in the design of the study, data interpretation, and helped draft the manuscript. JF critically assessed the manuscript and participated in the study discussion. SAL participated in the design of the study, data interpretation and study discussion. All authors read and approved the final manuscript.

\section{Author details}

${ }^{1}$ Department of Biomedical Physiology and Kinesiology, Simon Fraser University, 2600-515 West Hastings, Vancouver, British Columbia V6B 5K3, Canada. ${ }^{2}$ Department of Pathology and Laboratory Medicine, University of British Columbia, Vancouver, BC V6T 2B5, Canada. ${ }^{3}$ Division of Cardiology, Providence Health Care, Healthy Heart Program, 1081 Burrard Street,
Vancouver, BC V6Z 1Y6, Canada. ${ }^{4}$ Department of Medicine, University of British Columbia, 2775 Laurel Street, Vancouver, British Columbia V5Z 1M9, Canada. ${ }^{5}$ Faculty of Health Sciences, Simon Fraser University, Burnaby, British Columbia V5A 1S6, Canada.

Received: 24 April 2014 Accepted: 28 September 2014 Published: 10 October 2014

\section{References}

1. Ford ES, Li C, Sattar N: Metabolic syndrome and incident diabetes. Diabetes Care 2008, 31(9):1898-1904.

2. Gupta AK, Prieto-Merino D, Dahlof B, Sever PS, Poulter NR, ASCOT Investigators: Metabolic syndrome, impaired fasting glucose and obesity, as predictors of incident diabetes in 14120 hypertensive patients of ASCOT-BPLA: comparisons of their relative predictability using a novel approach. Diabetic Medicine 2011, 28(8):941-947.

3. Galassi A, Reynolds K, He J: Metabolic syndrome and risk of cardiovascular disease: a meta-analysis. Am J Med 2006, 119(10):812-819.

4. Gami AS, Witt BJ, Howard DE, Erwin PJ, Gami LA, Somers VK, Montori VM: Metabolic syndrome and risk of incident cardiovascular events and death: a systematic review and meta-analysis of longitudinal studies. JACC 2007, 49(4):403-414.

5. Mohan $\mathrm{V}$, Deepa M: The metabolic syndrome in developing countries. Diabetes Voice 2006, 51:15-17.

6. Ford ES, Giles WH, Mokdad AH: Increasing prevalence of the metabolic syndrome among US adults. Diabetes Care 2004, 27:2444-2449.

7. International Diabetes Federation: The IDF consensus worldwide definition of the metabolic syndrome. [https://www.idf.org/webdata/docs/ MetS_def_update2006.pdf]

8. Balkau B, Charles MA: Comment on the provisional report from the WHO consultation. European Group for the Study of Insulin Resistance (EGIR). Diabetes Medicine 1999, 16(5):442-443.

9. National Cholesterol Education Program (NCEP) Expert Panel on Detection, Evaluation, and Treatment of High Blood Cholesterol in Adults (Adult Treatment Panel III): Third report of the National Cholesterol Education Program (NCEP) Expert Panel on Detection, Evaluation, and Treatment of High Blood Cholesterol in Adults (Adult Treatment Panel III) Final Report. Circulation 2002, 106(25):3143.

10. Alberti KGMM, Eckel RH, Grundy SM, Grundy SM, Zimmet PZ, Cleeman I, Donato KA, Fruchart J-C, James PT, Loria CM, Smith SC Jr: Harmonizing the metabolic syndrome. A joint interim statement of the International Diabetes Federation Task Force on Epidemiology and Prevention; National Heart, Lung, and Blood Institute; American Heart Association; World Heart Federation; International Atherosclerosis Society; and International Association for the Study of Obesity. Circulation 2009, 120:1640-1645.

11. Gupta M, Singh N, Tsiqoulis M, Kaiij M, Hirjikaka S, Quan A, Teoh H, Verma S: Perceptions of Canadian primary care physicians towards cardiovascular risk assessment and lipid management. Can J Cardiology 2012, 28(1):14-19.

12. Cordero A, Laclaustra M, Leon M, Casasnovas JA, Grima A, Luengo E, Ordonez B, Bergua C, Bes M, Pascual I, Alegria E, on behalf of the MESYAS Registry Investigators: Comparison of serum lipid values in subjects with and without the metabolic syndrome. Am J Cardiol 2008, 102:424-428.

13. Onat A, Can G, Kaya H, Hergenc G: "Atherogenic index of plasma" ( $\log _{10}$ triglyceride/high-density lipoprotein-cholesterol) predicts high blood pressure, diabetes, and vascular events. J Clin Lipid 2010, 4:89-98.

14. Kimm H, Lee SW, Lee HS, Shim KW, Cho CY, Yun JE, Jee SH: Associations between lipid measures and metabolic syndrome, insulin resistance and adiponectin. Circ J 2010, 74:931-937.

15. Kawamoto R, Tabara Y, Kohara K, Miki T, Takayama S, Abe M, Katoh T, Ohtsuka N: Relationships between lipid profiles and metabolic syndrome, insulin resistance and serum high molecular adiponectin in Japanese community-dwelling adults. Lipids Health Dis 2011, 10:79.

16. Kim SW, Jee JH, Kim HJ, Jin SM, Suh S, Bae JC, Kim SW, Chung JH, Min YK, Lee MS, Lee MK, Kim KW, Kim JH: Non-HDL-cholesterol/HDL-cholesterol is a better predictor of metabolic syndrome and insulin resistance than apolipoprotein B/apolipoprotein A1. Int J Cardiol 2013, 168:2678-2683.

17. Arthur FKN, Adu-Frimpong M, Osei-Yeboah J, Mensah FO, Owusu L: Prediction of metabolic syndrome among postmenopausal Ghanian 
women using obesity and atherogenic markers. Lipids Health Dis 2012, 11:101.

18. Wang TD, Chen WJ, Chien KL, She-Yi Su SS, Hsu HC, Chen MF, Liau CS, Lee $\mathrm{YT}$ : Efficacy of cholesterol levels and ratios in predicting future coronary heart disease in a Chinese population. Am J Cardiol 2001, 88(7):737-743.

19. Ingelsson E, Schaefer $\mathrm{E}$, Contois $J H$, McNamara JR, Sullivan L, Keyes MJ, Pencina MJ, Schoonmaker C, Wilson PWF, D'Agostino RB, Vasan RS: Clinical utility of different lipid measures for prediction of coronary heart disease in men and women. JAMA 2007, 298(7):776-785.

20. McQueen MJ, Hawken S, Wang X, Ounpuu S, Sniderman A, Probstfield J, Steyn K, Sanderson JE, Hasani M, Volkova E, Kazmi K, Yusuf S, for the INTERHEART study investigators: Lipids, lipoproteins, and apolipoprteins as risk markers of myocardial infarction in 52 countries (the INTERHEART study): a case-control study. Lancet 2008, 372:224-233.

21. Eliasson B, Cederholm J, Eeq-Olofsson K, Svensson AM, Zethelius B, Gudbjornsdottir S, on behalf of the National Diabetes Register: Clinical usefulness of different lipid measures for prediction of coronary heart disease in type 2 diabetes. Diabetes Care 2011, 34(9):2095-2100.

22. Klein BEK, Klein R, Lee KE: Components of the metabolic syndrome and risk of cardiovascular disease and diabetes in Beaver Dam. Diabetes Care 2002, 25(10):1790-1794.

23. Hosmer DW, Lemeshow S: Applied Logistic Regression. 2nd edition. Hoboken, NJ: John Wiley \& sons, Inc; 2000:156-164.

24. Salazar MR, Carbajal HA, Espeche WG, Aizpurua M, Sisnieguez CE, March CE, Balbin E, Stavile RN, Reaven GM: Identifying cardiovascular disease risk and outcome: use of the plasma triglyceride/high-density lipoprotein cholesterol concentration ratio versus metabolic syndrome criteria. J Intern Med 2013, 273:595-601.

25. Vega GL, Barlow CE, Grundy SM, Leonard D, DeFina LF: Triglyceride-tohigh-density-lipoprotein-cholesterol ratio is an index of heart disease mortality and of incidence of type 2 diabetes mellitus in men. $J$ Investig Med 2014, 62:345-349.

26. Murquia-Romero M, Jimenez-Flores JR, Sinqrist-Flores SC, Espinoza-Camacho MA, Jimenez-Morales M, Pina E, Mendez-Cruz AR, Villalobos-Molina R, Reaven GM: Plasma triglyceride/HLD-cholesterol ratio, insulin resistance, and cardiometabolic risk in young adults. J Lipid Res 2013, 54(10):2795-2799.

27. Stein E, Kushner H, Gidding S, Falkner B: Plasma lipid concentrations in nondiabetic African American adults: association with insulin resistance and the metabolic syndrome. Metabolism 2007, 56(7):954-960.

28. Karelis AD, Pasternyk SM, Messier L, St-Pierre DH, Lavoie JM, Garrel D, Rabasa-Lhoret R: Relationship between insulin sensitivity and the triglyceride-HDL-C ratio in overweight and obese postmenopausal women: a MONET study. Appl Physiol Nutr Metab 2007, 32(6):1089-1096.

29. McLaughlin T, Reaven G, Abbasi F, Lamendola C, Saad M, Waters D, Simon J, Krauss RM: Is there a simple way to identify insulin-resistant individuals at increased risk of cardiovascular disease? Am J Cardiol 2005, 96(3):399-404

30. Fan X, Liu EY, Hoffman VP, Potts AJ, Sharma B, Henderson DC: Triglyceride/ high-density lipoprotein cholesterol ratio: a surrogate to predict insulin resistance and low-density lipoprotein particle size in nondiabetic patients with schizophrenia. J Clin Psych 2011, 72(6):806-812.

31. Kang H-T, Yoon J-H, Kim J-Y, Ahn S-K, Kinton JA, Koh S-B, Kim J-K: The association between the ratio of triglyceride to $\mathrm{HDL}-\mathrm{C}$ and insulin resistance according to waist circumference in rural Korean population. Nutr Metab Cardiovasc Dis 2012, 22(12):1054-1060.

32. Haedegh F, Hatami M, Tohidi M, Sarbakhsh P, Saadat N, Azizi F: Lipid ratios and appropriate cut off values for prediction of diabetes: a cohort of Iranian men and women. Lipids Health Dis 2010, 9:85.

33. Zoppini G, Negri C, Stoico V, Casati S, Pichiri I, Bonora E: Triglyceride-highdensity lipoprotein cholesterol is associated with microvascular complications in type 2 diabetes mellitus. Metabolism 2012, 61(1):22-29.

34. Jeppesen J, Hein HO, Suadicani P, Gyntelberg F: Relation of high TG-low $\mathrm{HDL}$ cholesterol and LDL cholesterol to the incidence of ischemic heart disease. An 8-year follow-up in the Copenhagen Male Study. Arterioscler Thromb Vasc Biol 1997, 17:1114-1120.

35. Hadaegh F, Khalili D, Ghasemi A, Tohidi M, Sheikholeslami F, Azizi F: Triglyceride/HDL-cholesterol ratio is an independent predictor for coronary heart disease in a population of Iranian men. Nutr Metab Cardiov Dis 2009, 19(6):401-408

36. Casella-Filho A, Hueb W, Jonke V, Santos R, Favarato D, Lima E, Segre A, Cury Rezende P, Larossa Garzillo C, Chacra APM, Ramires J, Kalil-Filho R: The
triglyceride/HDL ratio remains as an independent plasma lipid marker of major cardiovascular events in diabetic patients 10-year follow-up from the Medicine Angioplasty or Surgery Study II (MASS II). JACC 2012, 59(13s1):E1663-E1663 (abstract).

37. Salazar MR, Carbajal HA, Espeche WG, Aizpurua M, Maciel PM, Reaven GM: Identification of cardiometabolic risk: visceral adiposity index versus triglyceride/HDL cholesterol ratio. AM J Med 2014, 127:152-157.

38. Cordero A, Andres E, Ordonez B, Leon M, Laclaustra M, Grima A, Luengo E, Moreno J, Bes M, Pascual I, Civeira F, Pocovi M, Alegria E, Casasnovas JA, on behalf of the MESYAS (Metabolic Syndrome Active Subjects) Study investigators: Usefulness of triglycerides-to-high-density lipoprotein cholesterol ratio for predicting the first coronary event in men. Am J Cardiol 2009, 104:1393-1397.

39. Dobiasova M, Frohlich J: The plasma parameter log (TG/HDL-C) as an atherogenic index: correlation with lipoprotein particle size and esterification rate inapoB-lipoprotein-depleted plasma $\left(\mathrm{FER}_{\mathrm{HDL}}\right)$. Clin Biochem 2001, 34(7):583-588.

40. Li C, Ford ES, Menq YX, Mokdad AH, Reaven GM: Does the association of the triglyceride to high-density lipoprotein ratio with fasting serum insulin differ by race/ethnicity? Cardiovasc Diabetol 2008, 7:4.

41. Gasevic D, Frohlich J, Mancini GB, Lear SA: The association between triglyceride to high-density-lipoprotein cholesterol ration and insulin resistance in a multiethnic primary prevention cohort. Metabolism 2012, 61(4):583-589.

42. Sumner AE, Finley KB, Genovese DJ, Criqui MH, Boston RC: Fasting triglyceride and the triglyceride- $\mathrm{HDL}$ cholesterol ratio are not markers of insulin resistance in African Americans. Arch Int Med 2005, 165(12):1395-1400.

43. Knight MG, Goedecke JH, Ricks M, Evans J, Levitt NS, Tulloch-Reid MK, Sumner AE: The TG/HDL-C ratio does not predict insulin resistance in overweight women of African descent: a study of south African, African American, and west African women. Ethn Dis 2011, 21(4):490-494.

44. Lear SA, Humphries KH, Kohli S, Chockalingam A, Frohlich JJ, Birmingham $\mathrm{CL}$ : Visceral adipose tissue accumulation differs according to ethnic background: results of the Multicultural Community Health Assessment Trial (M-CHAT). Am J Clin Nutr 2007, 86(2):353-359.

45. Kohli S, Sniderman AD, Tchernof A, Lear SA: Ethnic-specific differences in abdominal subcutaneous tissue compartments. Obesity 2010, 18(11):2177-2183.

46. Kurian AK, Cardarelli KM: Racial and ethnic differences in cardiovascular disease risk factors: a systematic review. Ethn Dis 2007, 17(1):143-152.

47. Chiu M, Austin PC, Manuel DG, Tu JV: Comparison of cardiovascular risk profiles among ethnic groups using population health surveys between 1996 and 2007. CMAJ 2010, 182(8):E301-E310.

48. Lear SA, Birmingham CL, Chockalingam A, Humphries KH: Study design of the multicultural community health assessment trial (M-CHAT): A comparison of body fat distribution in four distinct populations. Ethn Dis 2006, 16:96-100

49. Friedwald WT, Levy Rl, Fredrickson DS: Estimation of the concentration of low-density lipoprotein cholesterol in plasma, without use of the preparative ultracentrifuge. Clin Chem 1972, 18:499-502.

50. Kriska AM, Knowler WC, LaPorte RE, Drash AL, Wing RR, Blair SN, Bennett PH, Kuller LH: Development of questionnaire to examine relationship of physical activity and diabetes in Pima Indians. Diabetes Care 1990, 13:401-411

51. Pereira MA, Kriska AM, Joswiak ML, Dowse GK, Collins VR, Zimmet PZ, Gareeboo H, Chitson P, Hemrai F, Purran A: Physical inactivity and glucose intolerance in the multiethnic island of Mauritius. Med Sci Sports Exerc 1995, 27:1626-1634.

52. Larsson B, Bengtsson C, Bjorntorp P, Lapidus L, Sjostrom L, Syardsudd K, Tibblin G, Wedel H, Welin L, Wilhelmsen L: Is abdominal body fat distribution a major explanation for the sex difference in the incidence of myocardial infarction? The study of men born in 1913 and the study ow women, Goteborg. Sweden. Am J Epidemiology 1992, 135(3):266-273.

53. Freedman DS, Jacobsen SJ, Barboriak JJ, Sobocinski KA, Anderson AJ, Kissebah AH, Sasse EA, Gruchow HW: Body fat distribution and male/ female differences in lipids and lipoproteins. Circulation 1990, 81:1498-1506

54. He H, Ni Y, Chen J, Zhao Z, Zhong J, Liu D, Yan Z, Zhang W, Zhu Z: Sex differences in cardiometabolic risk profile and adiponectin expression in subjects with visceral obesity. Trans/ Res 2010, 155(2):71-77. 
55. Youden WJ: Index for rating diagnostic tests. Cancer 1950, 3:32-35.

56. Schisterman EF, Perkins NJ, Liu A, Bondell H: Optimal cut-point and its corresponding Youden Index to discriminate individuals using pooled blood samples. Epidemiology 2005, 16:73-81.

57. DeLong ER, DeLong DM, Clarke-Pearson DL: Comparing the areas under two or more correlated receiver operating characteristic curves: a nonparametric approach. Biometrics 1988, 44:837-845.

doi:10.1186/1476-511X-13-159

Cite this article as: Gasevic et al: Clinical usefulness of lipid ratios to identify men and women with metabolic syndrome: a cross-sectional study. Lipids in Health and Disease 2014 13:159.

\section{Submit your next manuscript to BioMed Central and take full advantage of:}

- Convenient online submission

- Thorough peer review

- No space constraints or color figure charges

- Immediate publication on acceptance

- Inclusion in PubMed, CAS, Scopus and Google Scholar

- Research which is freely available for redistribution 